\title{
Fish consumption decreases, whereas meat consumption increases, dementia risk
}

Elderly people who eat fish regularly are $20 \%$ less likely to have dementia than those who do not eat fish at all, and this inverse association seems to be dosedependent, according to a populationbased study conducted in low-income and middle-income countries.

These findings confirm data from previous population-based studies, and are probably explained by the presence of long-chain omega-3 fatty acids in oily fish, as animal studies demonstrated that these molecules have anti-inflammatory,

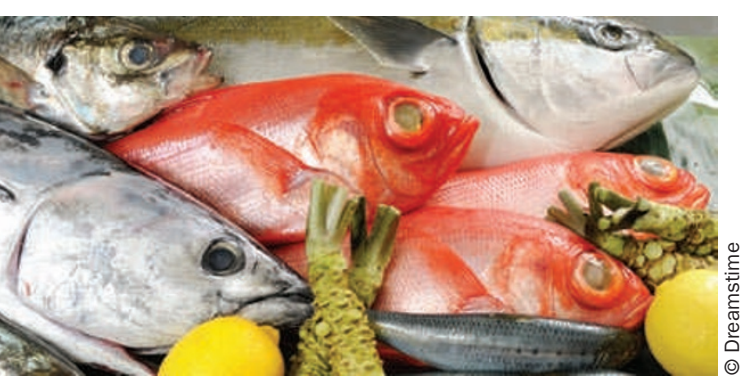

antioxidant, antiatherogenic, antiamyloid and neuroprotective properties.

To examine the relationships between fish and meat consumption and dementia, the investigators assessed 14,960 residents aged $\geq 65$ years in China, India, Cuba, the Dominican Republic, Venezuela, Mexico and Peru, using one-phase, population-based, cross-sectional surveys. Dementia was diagnosed according to validated criteria and dietary habits were ascertained in face-to-face interviews by trained interviewers.

At all sites except India, the investigators found that the risk of dementia was inversely associated with fish consumption and directly associated with meat consumption; both relationships were dose-dependent. "Our data have a relevant public health impact, also considering the rapid nutritional transition happening in these settings, and constitute an important replication of findings in very different and diverse settings" says Emiliano Albanese (King's College London, UK), lead investigator of the study.

Although $66 \%$ of people with dementia live in low-income and middle income countries, no more than $10 \%$ of population-based studies of dementia have been carried out in these regions. "Our collective of researchers, the 10/66 Dementia Research Group, aims to provide a detailed evidencebase to inform the development and implementation of policies for improving the health and social welfare of older people in low and middle income countries, particularly the 14 million people with dementia, their relatives and carers," says Albanese.

\section{Julianna Vig}

Original article Albanese, E. et al. Dietary fish and mea intake and dementia in Latin America, China, and India: a 10/66 Dementia Research Group population-based study. Am. J. Clin. Nutr. 90, 392-400 (2009). 